\title{
Parasitic Infection Surveillance in Mississippi Delta Children
}

\author{
Richard S. Bradbury, ${ }^{1,2}$ Irene Arguello, ${ }^{3}$ Meredith Lane, ${ }^{1}$ Gretchen Cooley, ${ }^{1}$ Sukwan Handali, ${ }^{1}$ Silvia D. Dimitrova, ${ }^{1}$ \\ Fernanda S. Nascimento, ${ }^{1}$ Sam Jameson, ${ }^{3}$ Kathryn Hellmann, ${ }^{3}$ Michelle Tharp, ${ }^{4,5}$ Paul Byers, ${ }^{6}$ Susan P. Montgomery, ${ }^{1}$ \\ Lisa Haynie, ${ }^{5}$ Brian Kirmse, ${ }^{4}$ Nils Pilotte, ${ }^{7,8}$ Steven A. Williams, ${ }^{7,8}$ and Charlotte V. Hobbs ${ }^{3,9 *}$ \\ ${ }^{1}$ Division of Parasitic Diseases and Malaria, Parasitic Diseases Branch, Center for Global Health, Centers for Disease Control and Prevention, \\ Atlanta, Georgia; ${ }^{2}$ School of Health and Life Sciences, Federation University, Berwick Campus, Melbourne, Australia; ${ }^{3}$ Department of Pediatrics, \\ Division of Infectious Diseases, Children's of Mississippi, Jackson, Mississippi; ${ }^{4}$ Division of Genetics, Department of Pediatrics, University of \\ Mississippi Medical Center, Jackson, Mississippi; ${ }^{5}$ The Delta Mercy Project, School of Nursing, University of Mississippi Medical Center, Jackson, \\ Mississippi; ${ }^{6}$ Mississippi State Department of Health, Jackson, Mississippi; ${ }^{7}$ Department of Biological Sciences, Smith College, Northampton, \\ Massachusetts; ${ }^{8}$ Program in Molecular and Cellular Biology, University of Massachusetts, Amherst, Massachusetts; ${ }^{9}$ Department of Microbiology, \\ University of Mississippi Medical Center, Jackson, Mississippi
}

\begin{abstract}
Some recent studies suggest ongoing transmission of parasitic diseases in the American South; however, surveys in Mississippi children are lacking. We enrolled 166 children (median age 8 years, range 4-13 years) from the Mississippi Delta region and carried out multi-parallel real-time polymerase chain reaction (PCR) for Necator americanus, Ascaris lumbricoides, and Strongyloides stercoralis on their stool samples. Dried blood spots were obtained for multiplex serology antibody detection. Of 166 children, all reported having flushable toilets, $11 \%$ had soil exposure, and $34 \%$ had a pet dog or cat. None had prior diagnosis or treatment of parasitic disease. Multi-parallel real-time PCRs were negative on the 89 stool DNA extracts available for testing. Dried blood spot testing of all 166 children determined the seroprevalence of IgG antibodies to Toxocara spp. (3.6\%), Cryptosporidium (2.4\%), S. stercoralis, Fasciola hepatica, and Giardia duodenalis (all 0\%). In conclusion, parasitic infections and exposure were scarce in this population. Larger studies of at-risk populations are needed.
\end{abstract}

\section{INTRODUCTION}

Hookworm and other soil-transmitted helminths (STHs) are diseases of poverty, which adversely affect the physical and cognitive development of children. ${ }^{1}$ In response to widespread hookworm STH infection in the Southeastern United States in the early twentieth century, the Rockefeller Sanitary Commission for the Eradication of Hookworm Disease was established in 1909. Over the following 5 years, the commission found that the prevalence of hookworm disease in Mississippi schoolchildren was $36.7 \% .{ }^{2}$ Following the eradication efforts and with improved sanitation and economic development, it was subsequently assumed that hookworm, and other STHs were unlikely to be a continued problem in the American South. ${ }^{1,3}$ However, the last comprehensive STH surveillance study in the Southeast was in $1975,{ }^{4}$ and STH cases continued to be reported to the Mississippi State Department of Health (MSDH) until the year 2000. The last report of hookworm to the MSDH was in 1992, when three cases were reported, as well as 11 cases of ascariasis and six of strongyloidiasis, although active reporting ceased in the preceding decade (B. Brackin, personal correspondence).

A 2017 study conducted in rural Alabama reported 19/55 and $4 / 55$ stool samples positive by real-time polymerase chain reaction (PCR) for Necator americanus and Strongyloides stercoralis, respectively. This has highlighted the possibility of continued foci of STH infection in the Southeastern United States. ${ }^{5}$ We conducted a pilot study to estimate the prevalence of STH and other potentially endemic parasitic infections of schoolchildren in the Mississippi Delta region. The present study specifically targeted children in Sharkey County, Mississippi, where the median per capita income was $\$ 15,430$ in 2016 . ${ }^{6}$

\footnotetext{
${ }^{*}$ Address correspondence to Charlotte V. Hobbs, Divisions of Infectious Disease and Microbiology, Department of Pediatrics, University of Mississippi Medical Center Batson Children's Hospital, 2500 North State St., Jackson, MS 39202. E-mail: chobbs@umc.edu
}

\section{METHODS}

Survey of schoolchildren. The survey of schoolchildren took place in Sharkey County, Mississippi, at a school-based health clinic run by the "Delta Mercy Project" (the University of Mississippi School of Nursing). At enrollment, children aged 2-18 years whose parent gave consent were eligible for enrollment; participating children older than 9 years provided assent. Basic history and physical examination were also collected/performed.

Within 1 year post-enrollment, on a rolling basis, and in conjunction with Delta Mercy Project health fair screenings/ school clinic visits, risk factor data including whether subjects resided in homes with flushable toilets, had animals (dog/cat), soil exposures, and prior diagnosis or treatment of parasitic disease were collected. In addition, one fresh fecal specimen per child was collected for microscopy and real-time PCR, and dried blood spots (DBS) were collected for antibody testing, but not contemporaneously. Stool samples were stored at $4^{\circ} \mathrm{C}$, transported, and processed in the laboratory within 24-72 hours post-collection.

Laboratory analysis. On receipt of fecal specimens in the laboratory at the University of Mississippi Medical Center (UMMC), two aliquots of $250 \mathrm{mg}$ were frozen for DNA extraction and storage. DNA was extracted using the SurePrep Soil DNA Isolation Kit (Fisher Scientific, Fair Lawn, NJ), with bead beating using zirconium beads (Benchmark, Sayreville, $\mathrm{NJ}$ ) for 3 minutes. Where sample volume was greater than $1 \mathrm{~g}$ direct microscopy was performed on fresh samples, as well as the saturated salt (specific gravity 1.2 ) centrifugal flotation. ${ }^{9}$

DNA extracts were stored at $-80^{\circ} \mathrm{C}$ until shipment on dry ice to $\mathrm{CDC}$ for real-time PCR analysis. At the CDC, samples were initially tested for inhibition using a novel human cytochrome $B$ gene real-time PCR (Supplemental Table 1). Non-inhibitory samples were then tested by multi-parallel real-time PCR for N. americanus, Ascaris lumbricoides, and S. stercoralis. ${ }^{8,9} \mathrm{~A}$ Ct value of $\leq 35$ was considered a positive result. Plasmid 
DNA-positive controls ${ }^{8}$ were used for each real-time PCR, and a PCR-grade water-negative control was incorporated into each run.

Dried blood spots (DBS) from all schoolchildren were collected on Whatman 903 filter paper (Cardiff, United Kingdom) and tested for IgG antibodies to Toxocara spp., ${ }^{10}$ S. stercoralis, ${ }^{11}$ Fasciola hepatica, ${ }^{12}$ Cryptosporidium parvum, and Giardia duodenalis ${ }^{13}$ using multiplex bead assays (MBAs) read via MAGPIX (Austin, TX) instruments to determine evidence of exposure to these diseases. All samples positive for Toxocara spp., S. stercoralis, or F. hepatica by multiplex serology were confirmed by Western blot. Full methods of MBA analysis are available in Supplemental Data.

Data storage/statistics. Data were logged in from case report forms and laboratory results into REDCap (https:// www.project-redcap.org), and Microsoft Excel (2016, Microsoft Corporation, Redmond, WA) was used for all calculations.

Ethics. This study was approved by the UMMC Institutional Review Board; on ethical review, the CDC was determined to be non-engaged. Permission was obtained from the school principal, and parents or guardians of children enrolled provided written permission for participation for the infants enrolled in this study. Children aged 9 years and older provided assent. All samples sent to the CDC were de-identified. No drug was administered as part of this observational study, but schoolchildren received standard of care management for illness from the Delta Mercy Health Care Clinic and UMMC medical personnel.

\section{RESULTS}

Overall, 166 children (median age 8 years, range 4-13 years) were enrolled in the study, including 75 males and 91 females. This study took place between July 2016 and August 2017.

No children reported prior parasitic disease diagnosis or treatment or living in a home with a non-flushable toilet. Onethird $(34 \%)$ of children reported having a pet cat or dog at home, whereas $11 \%$ reported soil exposure (walking barefoot or handling soil) (Table 1).

A total of 100 fecal samples were obtained. Only 16 fresh fecal samples from schoolchildren had sufficient volume to allow both DNA extraction and microscopy, and all of these microscopy results were negative. Eleven samples (11\%) were negative by the inhibition/extraction control testing and were not further tested. The remaining 89 samples were tested by multiparallel STH real-time PCR for $N$. americanus, $A$. lumbricoides, and $S$. stercoralis and found to be negative (Table 2).

All 166 children enrolled provided a DBS sample for multiplex serology testing. None of the samples tested positive for antibodies reacting with the S. stercoralis Ss-NIE-1 antigen or the combined G. duodenalis VSP3 and VSP5 antigens.

TABLE 1

Risk factors for soil-transmitted helminth and other parasitic infections as assessed by questionnaire of schoolchildren in Sharkey County, Mississippi, $2016(n=166)$

\begin{tabular}{lc}
\hline \multicolumn{1}{c}{ Risk factor } & Yes, $n(\%)$ \\
\hline No flushable toilets in the home & $0(0)$ \\
Soil exposure & $18(11)$ \\
Pets (dog/cat) & $56(34)$ \\
Prior diagnosis or treatment of intestinal parasite & $0(0)$ \\
\hline
\end{tabular}

TABLE 2

Results of multi-parallel real-time PCR for selected parasitic infections on fecal samples from schoolchildren in Sharkey county, Mississippi, 2016-2017 $(n=100)$

\begin{tabular}{lc}
\hline \multicolumn{1}{c}{ Multi-parallel real-time PCR } & Positive, $n(\%)$ \\
\hline Human cytochrome B gene & $89(89 \%)$ \\
Necator americanus & $0(0)$ \\
Strongyloides stercoralis & $0(0)$ \\
Ascaris lumbricoides & $0(0)$ \\
\hline PCR = polymerase chain reaction. & \\
${ }^{*}$ Extraction/inhibition control. &
\end{tabular}

Antibodies reacting with both the $C$. parvum $\mathrm{Cp} 17$ antigen (range 558-10,823 MFI) and with C. parvum Cp23 (range $331-16,451 \mathrm{MFI}$ ) were detected in four of 166 samples $(2.4 \%)$, which are required to determine a sample as positive. Antibodies to Toxocara spp. rTc-CTL-1 antigen (range 30-98 MFI) were detected in six of 166 samples (3.6\%). Antibodies reacting with the $F$. hepatica $\mathrm{rFh}-\mathrm{SAP} 2$ antigen $(76 \mathrm{MFI})$ were detected in one of 166 samples $(0.6 \%)$, but this sample tested negative in the $F$. hepatica confirmatory Western blot assay (Table 3).

\section{DISCUSSION}

We found a very low prevalence of parasitic diseases in children from this specific region of the Mississippi Delta. In this population, PCR and limited microscopy data suggest a lack of transmission of the important STH species in question: $N$. americanus, $A$. lumbricoides, and S. stercoralis.

Our risk factor data also suggested that most children enrolled did not have risk factors that would increase their likelihood of STH infection. These results may be subject to bias as certainly there is a stigma attached to the questions regarding soil exposure and the type of toilet in the house. Poverty is closely linked to a number of these infections, ${ }^{4,14}$ and the children enrolled came from a county in which the average income is below the poverty line cutoff for a household of four. ${ }^{6}$

The seroprevalence of Toxocara spp. in this school-based study is within the range of estimated prevalences in the agegroups of 6-11 (3.0\%) and 12-19 (3.9\%) years that were recently published in a larger national study. ${ }^{15}$ With respect to Cryptosporidium, the $2.4 \%$ prevalence in this small study of school-aged children is lower than in previous reports, although the comparison data are older and our study includes slightly younger children. ${ }^{15}$ The seroprevalence of Giardia was zero in this study, suggesting no recent exposure.

Our PCR and limited microscopy data suggest the absence of $N$. americanus, $A$. lumbricoides, and $S$. stercoralis infection

TABLE 3

Seroprevalence rates for selected parasitic infections in dried blood spots collected from schoolchildren in Sharkey County, Mississippi, 2016-2017 $(n=166)$

\begin{tabular}{lc}
\hline \multicolumn{1}{c}{ Multiplex serology } & Positive, $n(\%)$ \\
\hline Toxocara species & $6(3.6)$ \\
Strongyloides stercoralis & $0(0)$ \\
Fasciola hepatica & $0(0)^{\star}$ \\
Cryptosporidium parvum & $4(2.4)$ \\
Giardia duodenalis & $0(0)$ \\
\hline *One sample positive by MAGPIX multiplex serology was found to be negative by the \\
confirmatory Western blot test.
\end{tabular}


in this population. It should be considered that Sharkey County had a historically low prevalence of $N$. americanus infection, with the Rockefeller Sanitary Commission reporting no more than $5.0 \%$ prevalence. ${ }^{16}$ The slowly permeable clay alluvium soils of Sharkey County are not generally conducive to hookworm development, which is best achieved in aerated sandy or loamy soil. ${ }^{17-19}$

Of note, because we concluded that our PCR results were negative and because PCR is not the current standard of care test used for diagnosis of STH infections, no children were referred for treatment. In the absence of signs and symptoms due to infection, the positive results for Toxocara and intestinal protozoa antibodies likely indicated exposure only and were not indication for treatment referral. Results for this study have been disseminated back to the community. All data (deidentified) have been communicated to the Mississippi Department of Health.

In comparison to a recent study performed in Lowndes County, Alabama, our results indicate negligible transmission of STHs and intestinal protozoa among children from Sharkey County, Mississippi. However, the possibility that isolated foci of STH infection remaining in marginalized communities that have poor sanitation and hygiene and poor access to health care, as observed in other developed countries such as Australia, ${ }^{20}$ cannot be excluded by the data presented here. Further investigation targeting counties specifically chosen for the aforementioned risk factors, as well as testing of additional age-groups, is warranted.

Received January 11, 2020. Accepted for publication April 12, 2020.

Published online June 22, 2020.

Note: Supplemental table appears at www.ajtmh.org.

Acknowledgments: We thank the children and parents of the Mississippi Delta for their participation in this study. We thank Sarah Sapp of the Centers for Disease Control and Prevention for her critical review of this article. We thank Bruce Brackin, Mississippi Department of Health, for historical data review from Mississippi Department of Health Archives.

Disclosure: This work was funded by the Centers for Disease Control and Prevention (RSB), the Blakeslee Fund for Genetics Research at Smith College (N. P. and S. A. W.), and the University of Mississippi Medical Center (Office of the Vice Chancellor for Research, the University of Mississippi Medical Center). R. B. reports a patent WO2019060840 "Removing Interfering Host NucleicAcids for Molecular Parasite Detection" with royalties paid to Centers for Disease Control and Prevention. This trial was observational and is exempt from registration at clinicaltrials.gov

Disclaimer: The findings and conclusions of this work are those of the authors and do not necessarily represent the official position of the Centers for Disease Control and Prevention/the Agency for Toxic Substances and Disease Registry. This work was presented at the American Society of Tropical Medicine and Hygiene Conference: Poster 522, October 28-November 1, New Orleans, LA.

Authors' addresses: Richard S. Bradbury, Division of Parasitic Diseases and Malaria, Parasitic Diseases Branch, Center for Global Health, Centers for Disease Control and Prevention, Atlanta, GA, and Federation University, Berwick Campus, Melbourne, Australia, E-mail: r.bradbury@federation.edu.au. Irene Arguello, Sam Jameson, Kathryn Hellmann, and Charlotte V. Hobbs, Divisions of Infectious Disease and Microbiology, Department of Pediatrics, University of Mississippi Medical Center, Jackson, MS, E-mails: iarguello@umc.edu, sjameson@ umc.edu, khellmann@umc.edu, and chobbs@umc.edu. Meredith Lane, Gretchen Cooley, Sukwan Handali, Silvia D. Dimitrova, Fernanda S. Nascimento, Susan P. Montgomery, Division of Parasitic Diseases and Malaria, Parasitic Diseases Branch, Center for Global Health,
Centers for Disease Control and Prevention, Atlanta, GA, E-mails: oeq5@cdc.gov, xxd1@cdc.gov, ahi0@cdc.gov, nwk2@cdc.gov, fs nascimento@yahoo.com.br, and zqu6@cdc.gov. Michelle Tharp, Division of Genetics, Department of Pediatrics, University of Mississippi Medical Center, Jackson, MS, and The Delta Mercy Project, School of Nursing, University of Mississippi Medical Center, Jackson, MS, E-mail: mtharp2@umc.edu. Paul Byers, Mississippi State Department of Health, Jackson, MS, E-mails: paul.byers@ msdh.ms.gov or pbyers@msdh.ms.gov. Lisa Haynie, The Delta Mercy Project, School of Nursing, University of Mississippi Medical Center, Jackson, MS, E-mail: Ihaynie@umc.edu. Brian Kirmse, Division of Genetics, Department of Pediatrics, University of Mississippi Medical Center, Jackson, MS, E-mail: bkirmse@umc.edu. Nils Pilotte and Steven A. Williams, Department of Biological Sciences, Smith College, Northampton, MA, and Program in Molecular and Cellular Biology, University of Massachusetts, Amherst, MA E-mails: npilotte@smith.edu and swilliam@smith.edu.

This is an open-access article distributed under the terms of the Creative Commons Attribution (CC-BY) License, which permits unrestricted use, distribution, and reproduction in any medium, provided the original author and source are credited.

\section{REFERENCES}

1. Hotez PJ, Brindley PJ, Bethony JM, King $\mathrm{CH}$, Pearce $\mathrm{EJ}$, Jacobson J, 2008. Helminth infections: the great neglected tropical diseases. $J$ Clin Invest 118: 1311-1321.

2. Elman C, McGuire RA, Wittman B, 2014. Extending public health: the Rockefeller Sanitary Commission and hookworm in the American south. Am J Public Health 104: 47-58.

3. Humphreys $M, 2009$. How four once common diseases were eliminated from the American South. Health Aff (Millwood) 28: $1734-1744$.

4. Starr MC, Montgomery SP, 2011. Soil-transmitted helminthiasis in the United States: a systematic review-1940-2010. Am J Trop Med Hyg 85: 680-684.

5. McKenna ML, McAtee S, Bryan PE, Jeun R, Ward T, Kraus J, Bottazzi ME, Hotez PJ, Flowers CC, Mejia R, 2017. Human intestinal parasite burden and poor sanitation in rural Alabama. Am J Trop Med Hyg 97: 1623-1628.

6. Bureau USC, 2017. Quick Facts: Sharkey County, Mississippi; Mississippi. Available at: https://www.census.gov/quickfacts/ fact/table/sharkeycountymississippi,MS/PST045219. Accessed October 13, 2018.

7. Gillespie S, Bradbury RS, 2017. A survey of intestinal parasites of domestic dogs in central Queensland. Trop Med Infect Dis 2: 60 .

8. Pilotte N, Papaiakovou M, Grant JR, Bierwert LA, Llewellyn S, McCarthy JS, Williams SA, 2016. Improved PCR-based detection of soil transmitted helminth infections using a nextgeneration sequencing approach to assay design. PLoS Negl Trop Dis 10: e0004578.

9. Pilotte N, Maasch J, Easton AV, Dahlstrom E, Nutman TB, Williams SA, 2019. Targeting a highly repeated germline DNA sequence for improved real-time PCR-based detection of Ascaris infection in human stool. PLoS Negl Trop Dis 13: e0007593.

10. Anderson JP et al., 2015. Development of a luminex bead based assay for diagnosis of toxocariasis using recombinant antigens Tc-CTL-1 and Tc-TES-26. PLoS Negl Trop Dis 9: e0004168.

11. Rascoe LN, Price C, Shin SH, McAuliffe I, Priest JW, Handali S, 2015. Development of Ss-NIE-1 recombinant antigen based assays for immunodiagnosis of strongyloidiasis. PLoS Negl Trop Dis 9: e0003694.

12. Shin SH, Hsu A, Chastain HM, Cruz LA, Elder ES, Sapp SG, McAuliffe I, Espino AM, Handali S, 2016. Development of two FhSAP2 recombinant-based assays for immunodiagnosis of human chronic fascioliasis. Am J Trop Med Hyg 95: 852-855.

13. Priest JW, Moss DM, Visvesvara GS, Jones CC, Li A, Isaac-Renton $\mathrm{JL}, 2010$. Multiplex assay detection of immunoglobulin $\mathrm{G}$ antibodies that recognize Giardia intestinalis and Cryptosporidium parvum antigens. Clin Vaccine Immunol 17: 1695-1707.

14. Becker DJ, Oloya J, Ezeamama AE, 2015. Household socioeconomic and demographic correlates of Cryptosporidium 
seropositivity in the United States. PLoS Negl Trop Dis 9: e0004080.

15. Liu EW, Chastain HM, Shin SH, Wiegand RE, Kruszon-Moran D, Handali S, Jones JL, 2018. Seroprevalence of antibodies to Toxocara species in the United States and associated risk factors, 2011-2014. Clin Infect Dis 66: 206-212.

16. Eric T, 2009. Historic Hookworm Prevalence Rates and Distribution in the Southeastern United States: Selected Findings of the Rockefeller Sanitary Commission for the Eradication of Hookworm. Available at: https://pdfs.semanticscholar.org/ 1ae1/97ea8482ffb91ad41410924e7d6920381485.pdf?_ga= 2.172215587.1469608792.1569007518-2001121332.1569 007518. Accessed May, 2018.
17. Berry SH, Pevar J, Zander-Cotugno M, 2008. Use of Incentives in Surveys Supported by Federal Grants (Rand Corporation). Available at: http://www.copafs.org/seminars/use_of_incentives_ in_surveys.aspx. Accessed June 5, 2019.

18. Jourdan PM, Lamberton PHL, Fenwick A, Addiss DG, 2017. Soiltransmitted helminth infections. Lancet 391: 252-265.

19. Project MSE, 2018. Introduction to Soil Types, Mississippi. Available at: http://extension.msstate.edu/agriculture/crops/ soils/introduction-soils. Accessed April 10, 2018.

20. Gordon CA, Kurscheid J, Jones MK, Gray DJ, McManus DP, 2017. Soil-transmitted helminths in tropical Australia and Asia. Trop Med Infect Dis 2: 56. 